\title{
Fourier Transform Infrared Characterization of the Azido Complex of Methane Monooxygenase Hydroxylase from Methylococcus capsulatus (Bath)
}

\author{
Shen Lu, ${ }^{\ddagger}$ Matthew H. Sazinsky, ${ }^{\dagger}$ James W. Whittaker, ${ }^{\ddagger}$ Stephen J. Lippard, ${ }^{\dagger}{ }^{\dagger}$ and Pierre Moënne- \\ LocCOZ $^{* \ddagger}$ \\ Department of Environmental \& Biomolecular Systems, OGI School of Science \& Engineering, Oregon Health \& \\ Science University, Beaverton, Oregon, 97006-8921, and Department of Chemistry, Massachusetts Institute of \\ Technology, Cambridge, Massachusetts 02139
}

\section{Supporting Information:}

\section{Experimental Details:}

Multiple attempts were made to determine the crystal structure of an $\mathrm{MMOH}$ azide complex. Oxidized $\mathrm{MMOH}$ crystals were grown in the presence of $5 \mathrm{mM}$ sodium azide and then soaked with $>100 \mathrm{mM}$ sodium azide before data collection. In all cases the soaked crystals turned bright yellow, indicative of binding of azide to the diiron centers. However, every structure determined to $\sim 2.0 \AA$ resolution revealed no features near the diiron centers that could be assigned to azide (water molecules and/or formate were best modeled into the vicinity of the diiron centers). Most likely, under these crystallization and soaking conditions, the occupancy of azide at the diiron center was too low for clear structural assignment, or there was positional disorder in the crystal. Attempts to increase the azide concentration $>200 \mathrm{mM}$ cracked the crystals.

For FTIR experiments, the azido complex of FeSOD was prepared at room temperature using a $1.5 \mathrm{mM}$ enzyme concentration ( $3 \mathrm{mM}$ in monomer) in $50 \mathrm{mM}$ phosphate $\mathrm{pH} 7.0$ and $10 \mathrm{mM}$ sodium azide. The extent of azido complex formation was estimated to be $35 \%$ by comparing the UV-vis absorption increase at $430 \mathrm{~nm}$ with that observed in saturating conditions (i.e. $100 \mathrm{mM}$ azide). In the $\mathrm{MMOH}$ experiments, the enzyme concentration was $\sim 1 \mathrm{mM}$ in $25 \mathrm{mM}$ MOPS pH 7.0 and $50 \mathrm{mM}$ azide. Comparison of the UV-vis absorption change with that observed in saturating conditions $(500 \mathrm{mM})$ suggest that $\sim 60 \%$ of azido complex formation was in the FTIR sample.

A $11 \mu \mathrm{L}$ droplet of the azido complex was sandwiched between two $\mathrm{CaF}_{2}$ windows with a $15-\mu \mathrm{m}$ Teflon spacer. A Cary 50 spectrometer was used to obtain a UV-vis spectrum of the sample in the FTIR cell before mounting it to a closed-cycle cryogenic system (Displex, Advanced Research Systems) (Fig. S1). The cryostat was installed in the FTIR sample compartment and kept in the dark while the temperature dropped to $15 \mathrm{~K}$. Series of FTIR spectra of 1000-scan accumulation were collected with a 4-cm ${ }^{-1}$ resolution using a Perkin-Elmer system 2000 equipped with a liquid- $\mathrm{N}_{2}$ cooled MCT detector.

Photolysis was induced by continuous illumination of the sample in the FTIR sample chamber using a $50 \mathrm{~W}$ tungsten lamp after filtering out heat and NIR emission. The amplitude of the FTIR difference spectra ("dark" illuminated") reached a plateau after Illumination period ranging from 15-20 min. The temperature of the sample was monitored and controlled with a Cry-Con 32 unit.

The percentage of photolyzed population which could be trapped at $15 \mathrm{~K}$ was deduced from curve fitting analyses (Fig. S3). For FeSOD-N 3 , the dark spectrum was curve fitted with two Gaussian curves: one at 2045 $\mathrm{cm}^{-1}$ (free azide) and one at $2056 \mathrm{~cm}^{-1}$ that corresponds to the iron-azido complex. The positive signal in the "dark" minus "illuminated" spectrum represents $17 \pm 5 \%$ of the signal observed at $2056 \mathrm{~cm}^{-1}$. In $\mathrm{MMOH}-\mathrm{N}_{3}$, the contribution of free azide is so large that the total concentration of azido complex formed cannot be determined from the dark FTIR spectrum at $15 \mathrm{~K}$. Using the UV-vis absorption at room temperature and assuming no severe change in binding affinity as the temperature is lower to $15 \mathrm{~K}$, the photolysis is estimated at $15 \pm 10 \%$. The reversibility of the photolysis process was confirmed by FTIR and UV-vis absorption (Fig. S4 and S5). 
Figure S1. Room temperature UV-Vis absorption spectra of MMOH-N ${ }_{3}$ (red) and FeSOD-N $\mathrm{N}_{3}$ (blue) obtained directly in the FTIR cell (15 $\mu \mathrm{m}$ pathlength). The spectrum of FeSOD$\mathrm{N}_{3}$ was divided by 3 to facilitate the comparison of the two traces.

Figure S2. Room temperature resonance Raman spectra of oxidized $\mathrm{MMOH}$ (black trace), $\mathrm{MMOH}-\mathrm{N}_{3}$ (red trace) and MMOH $-{ }^{15} \mathrm{~N}^{14} \mathrm{~N}_{2}$ (blue trace) obtained with $458-\mathrm{nm}$ excitation. The enzyme concentration was $1.3 \mathrm{mM}$ and the total azide concentration was $500 \mathrm{mM}$. A weak and broad signal at $\sim 370 \mathrm{~cm}^{-1}$ is observed in the spectra of the azido complexes but absent in the spectrum of oxidized $\mathrm{MMOH}$ and is tentatively assigned to a $v_{\text {as }}\left(\mathrm{Fe}-\mathrm{N}_{3}\right)$. The envelope of this band appears to shift to low frequency as the azido complex is formed with ${ }^{15} \mathrm{NN}_{2}$, but a definitive assignment would require experiments with ${ }^{15} \mathrm{~N}_{3}$-labeling.

Figure S3. FTIR spectra of FeSOD-N $\mathrm{N}_{3}$ obtained at $15 \mathrm{~K}$. The top trace (red) is a "dark" spectrum of FeSOD-N ${ }_{3}$ showing the iron bound azide $\left(2056 \mathrm{~cm}^{-1}\right)$ and excess azide in solution $\left(2045 \mathrm{~cm}^{-1}\right)$. The overlapping curve fit (dotted black trace), is obtained with two Gaussian components (green and blue solid traces). The residual from the fit is also shown (black trace). The lower trace corresponds to the "dark" minus "illuminated" FTIR difference spectrum obtained with the same sample (blue trace) and has been multiplied by 6 .
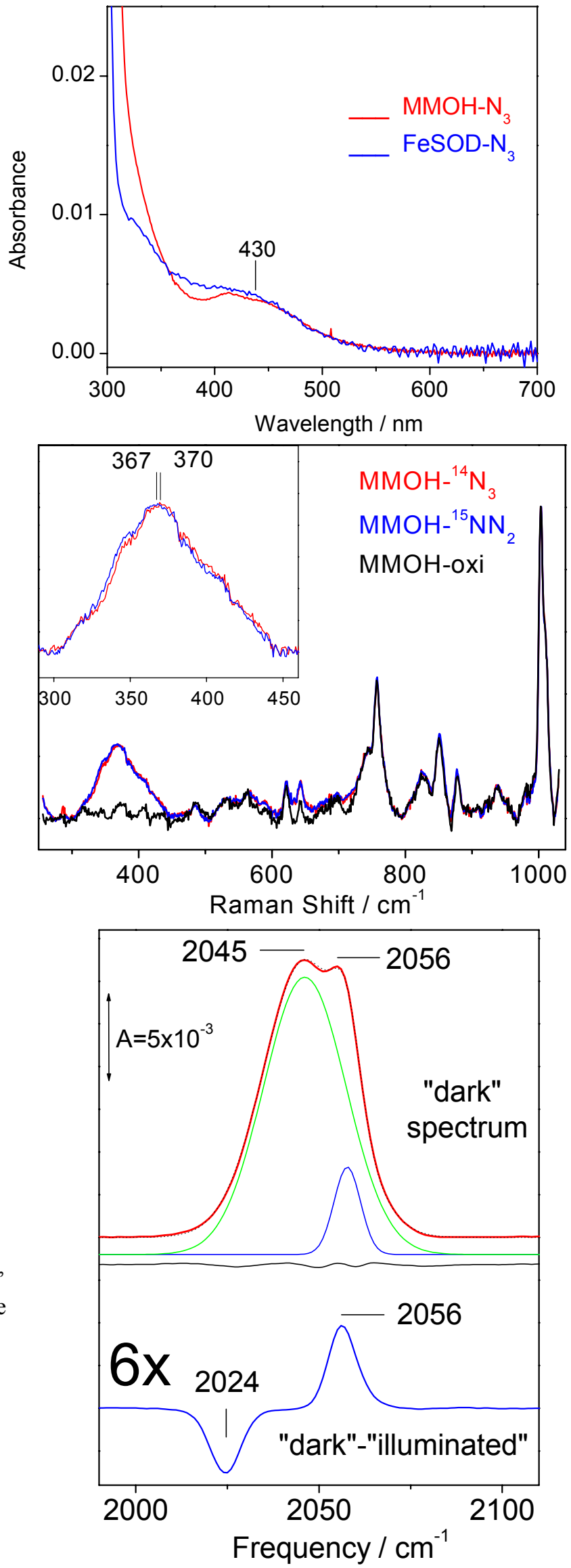
Figure S4. UV-Vis absorption spectra of $\mathrm{MMOH}-\mathrm{N}_{3}$ obtained directly in the FTIR cell (15 $\mu \mathrm{m}$ pathlength) before and after the low temperature FTIR experiments.

Figure S5. FTIR difference spectra of $\mathrm{MMOH}-\mathrm{N}_{3}$ obtained at $15 \mathrm{~K}$. The top trace (red) is a first "dark" minus "illuminated" difference spectrum. The second trace (blue) is a new "dark" minus "illuminated" difference spectrum obtained after an annealing period during which the temperature of the sample has been raised above $200 \mathrm{~K}$. The bottom trace (green) is the difference spectrum between the two dark spectra and confirms the total reversibility of the photolytic process.
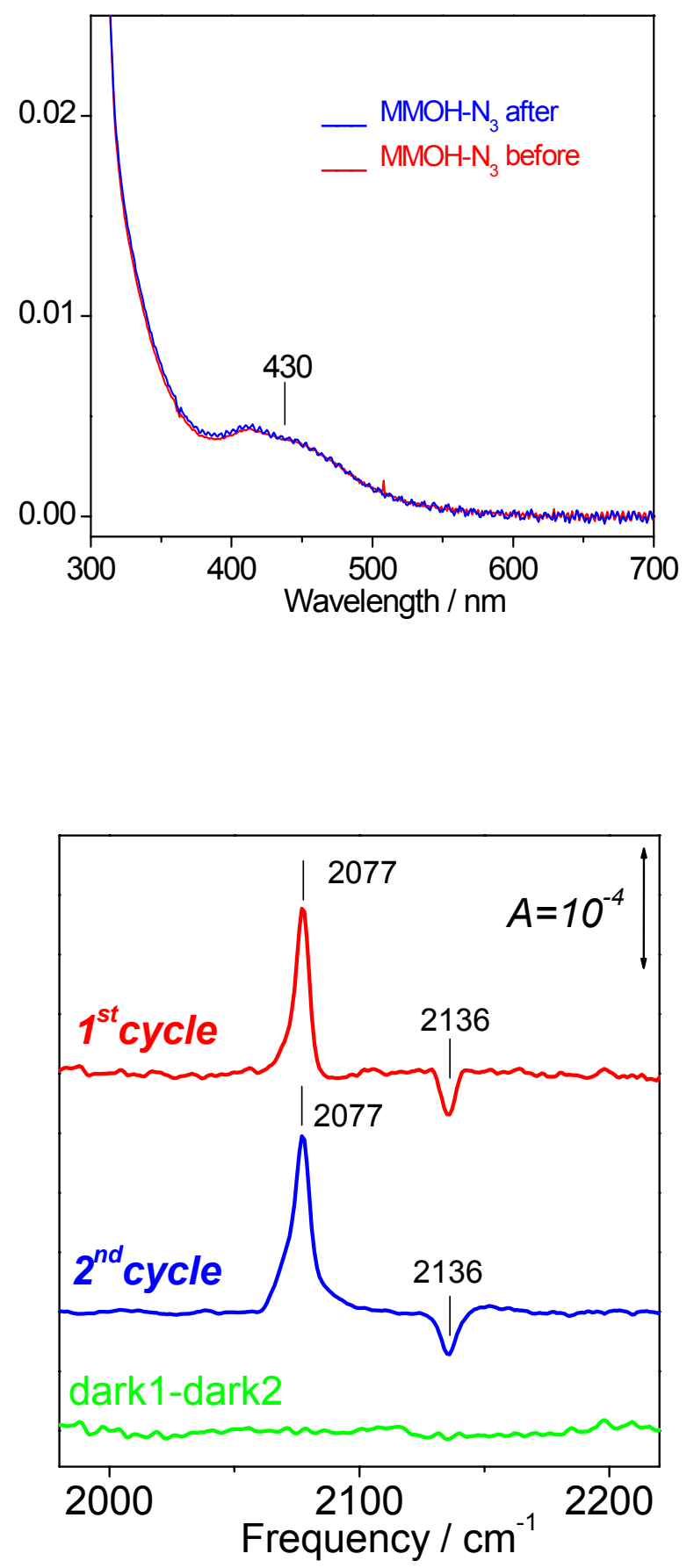